\title{
The Use of Spatial Stream Network Models to Evaluate the Effects of Varying Stream Temperatures on Wild Coho Life History Expression and Survival
}

\author{
Marisa N.C. Litz, Mickey Agha, John J. Winkowski, Devin West, and Jennifer Kordosky \\ Washington Department of Fish and Wildlife, PO Box 43200, Olympia, WA 98504-3200, USA
}

Keywords: coho salmon, jacks, climate change, forecasts, freshwater temperature, marine indicators

Climate change is affecting survival of Pacific salmon (Oncorhynchus spp.) stocks all along the west coast of North America. In this study, we evaluated survival of wild coho salmon (O. kisutch) measured at Bingham Creek in western Washington, where a full census trap has operated since 1982 (Fig. 1). Annually at the trapping site, all coho smolts were marked with a coded wire tag (CWT) and released. Returns of tagged precocious males (jacks) and recoveries of tagged adults in fisheries and returning to the trap were used to estimate marine survival. The relationship between jack and adult marine survival has been used as a management tool to forecast returns of wild coho throughout coastal watersheds for decades but has become increasingly unreliable. The goal of this study was to use environmental indicators to better account for variation in jack and adult marine survival and improve forecasting skills.

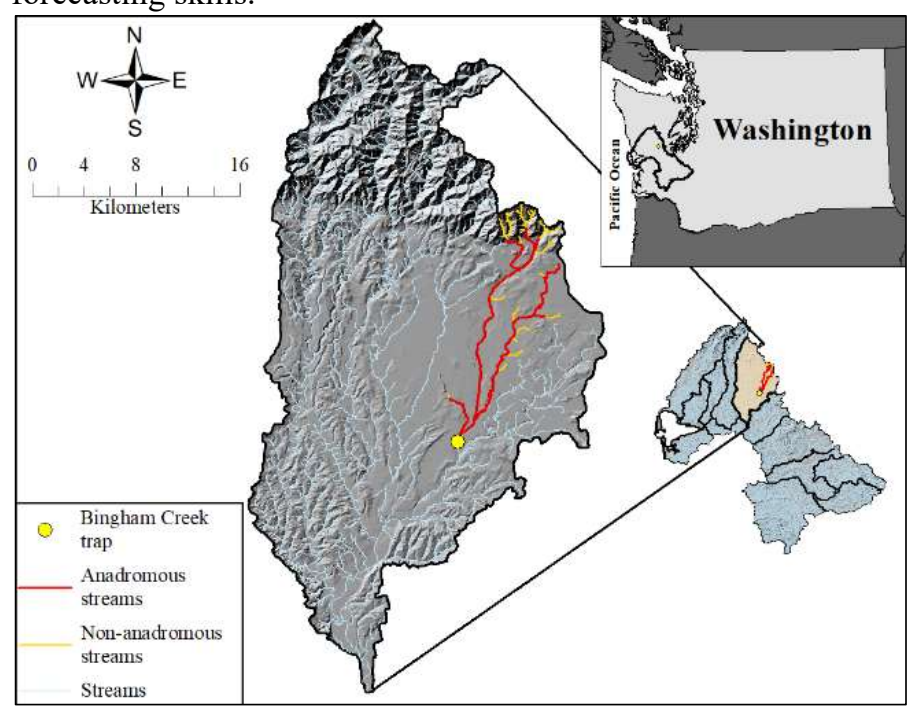

Fig. 1. Bingham Creek trap location showing anadromous and non-anadromous streams upstream of the trap location. Map credit: E. Walther, Washington Department of Fish and Wildlife.
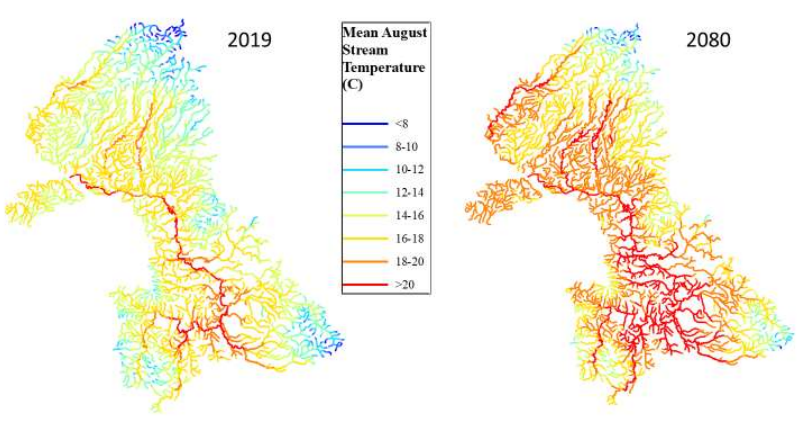

Fig. 2. Maps showing mean August stream temperature in the Chehalis basin in 2019 and 2080.

Wild coho jack rates for brood years 1980 to 2016 were evaluated over time and found to be increasing, especially after 2005 , a year characterized by delayed upwelling and low productivity in the coastal environment (Brodeur et al. 2006; Schwing et al. 2006). Jacking is thought to be initiated by reaching a specific growth or body condition threshold by a certain point in the salmon life cycle (DeFilippo et al. 2019), although it is uncertain whether this occurs in freshwater or during the early marine stage. Freshwater rearing conditions can influence smolt characteristics such as emigration timing and body size, which in turn influence age-at-maturity (jack rates) in the marine environment. We hypothesized that jack rates were related to freshwater rearing conditions (e.g., streamflow, temperature) and that by accounting for variation in stream flow and temperature during critical life 
history periods, we could better predict jack abundance and improve forecast model performance (Fig. 2). We also considered the role of ocean conditions during the first few months following freshwater emigration.

Table 1. Freshwater indicators used to predict coho salmon jack rate at the Bingham Creek trap (brood years 1996 to 2016). Indicators included spawner abundance (adults passed above the Bingham Creek trap) and cumulative, minimum, and maximum (cum., min., max.) stream flow during life history phases measured at the Satsop River (USGS 1203500). Stream temperatures for missing dates over the time series were derived from the relationship between stream flow and stream temperature measured at the Satsop River (Ecology 22G070) from 1960 to 1993.

\begin{tabular}{llll}
\hline Freshwater Indicators & Period & Metric & Source \\
\hline Spawner Abundance & Spawning (Oct-Dec) & cum., min., max. & Bingham Creek trap \\
Stream Flow & Spawning (Oct-Dec) & cum., min., max. & waterdata.usgs.gov \\
& Incubation (Dec-Feb) & cum., min., max. & waterdata.usgs.gov \\
& Summer Rearing (Aug) & cum., min., max. & waterdata.usgs.gov \\
& Overwinter (Nov-Feb) & cum., min., max. & waterdata.usgs.gov \\
Stream Temperature & Summer Rearing (Aug) & mean & apps.ecology.wa.gov \\
\hline
\end{tabular}

The freshwater dataset used to evaluate variation in jack rates through time (brood years 1996 to 2016) included spawner abundance, stream flow during spawning, incubation, summer rearing, overwinter periods, and stream temperature during the peak summer rearing period (Table 1). In addition, spatially continuous mean August stream temperature maps ("Thermalscapes") were developed for current conditions and mid- (2040) and late-century (2080) stream temperature scenarios based on climate change projections (Winkowski et al. 2018; Winkowski and Zimmerman 2019). The spatial stream network (SSN) models used in this study fit temperature data measured at 123 new monitoring stations since 2014 in addition to the 36 sites used in the NorWeST summer stream temperature model (Isaak et al. 2014, 2017).

Table 2. Marine indicators used to predict jack rate and adult marine survival of coho salmon at the Bingham Creek trap (brood years 1996 to 2016). Indicators from the basin scale included thermal regime (cool $=1996$ to 1999 plus La Niña years and warm = 2000 to 2016 plus El Niño years), North Pacific Gyre Oscillation (NPGO), Aleutian Low-Beaufort Sea Anticyclone (ALBSA), Pacific Decadal Oscillation (PDO), and Oceanic Niño Index $(\mathrm{ONI})$. Regional indicators included timing of the physical spring transition from predominantly downwelling to upwelling conditions based on hydrography, sea surface temperature, deep salinity, zooplankton, and ichthyoplankton measurements off Newport, Oregon (fisheries.noaa.gov/west-coast/science-data/oceanecosystem-indicators-pacific-salmon-marine-survival-northern). The local indicator was Chehalis River flow (USGS 12027500) during smolt outmigration.

\begin{tabular}{llll}
\hline Marine Indicators & Scale & Period & Source \\
\hline Thermal Regime & Basin & Warm/Cool & Based on sibling relationships \\
NPGO & Basin & May-Sep & o3d.org/npgo/enso.html \\
ALBSA & Basin & May-Sep & psl.noaa.gov/data/timeseries/ALBSA/ \\
PDO & Basin & May-Sep & NOAA Stoplight Table \\
ONI & Basin & Jan-Jun & NOAA Stoplight Table \\
Physical Spring Transition & Regional & Spring & NOAA Stoplight Table \\
SST NDBC Buoys & Regional & May-Sep & NOAA Stoplight Table \\
Deep Salinity & Regional & May-Sep & NOAA Stoplight Table \\
Zooplankton & Regional & May-Sep & NOAA Stoplight Table \\
Ichthyoplankton & Regional & Jan-Mar & NOAA Stoplight Table \\
Outmigration Flow & Local & Apr-Jun & waterdata.usgs.gov \\
\hline
\end{tabular}

Marine indicators used to evaluate jack and adult marine survival rates integrated environmental information across multiple spatial scales and included outmigration stream flow, timing of the spring transition from downwelling to upwelling conditions, sea surface temperature, salinity, zooplankton, ichthyoplankton, North Pacific Gyre Oscillation (NPGO), Aleutian Low-Beaufort Sea Anticyclone (ALBSA), Pacific Decadal Oscillation (PDO), 
and Oceanic Niño Index (ONI) measurements during the early marine residence period (January through September of ocean entry year) for brood years 1996 to 2016 (Table 2). Generalized additive mixed models (GAMMs) were used to model jack and adult return rates over time and to model jack and adult marine survival as a function of environmental indicators using the R statistical package v.3.6.1 (R Core Team 2019) and mgcv package (Wood 2017).

All GAMMs were fitted with an autoregressive corAR1 error structure for correlation from one brood year to the next, a gaussian distribution with an identity link function, and included a fixed effect for thermal regime. Models were fitted with thin plate regression splines for parameter components, and the dimensions or degrees of freedom (i.e., knots, or degree of smoothing) were restricted for the fixed year parameters to a maximum of 5 to prevent overfitting. Support for candidate models was determined using $\mathrm{AIC}_{\mathrm{c}}$ based on a full subsets approach (Sobocinski et al. 2021) where all covariates were assessed for collinearity and candidate models did not include covariates that were correlated with one another $(r>0.28)$. The predictive performance of the top-ranked model was assessed using a one-year ahead approach and compared to observed values with recent five-year model evaluation statistics.
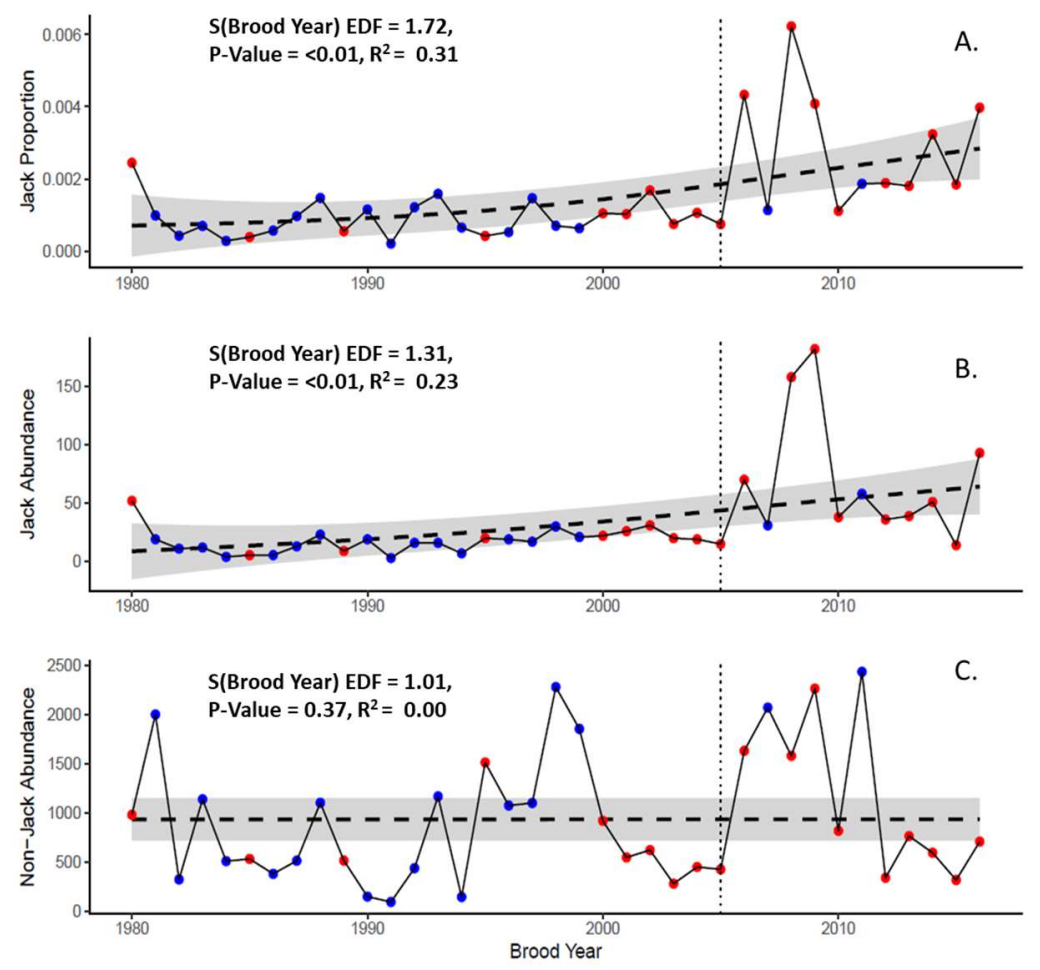

Fig. 3. Temporal trends in a) jack rate, b) jack abundance, and c) non-jack (i.e., adult) returns to Bingham Creek (brood years 1980 through 2016) with a breakpoint identified in 2005. Colored points correspond with warm (red) and cool (blue) regimes. A smoothing function (dashed black line and gray envelope showing 95\% confidence intervals have been added to illustrate general trends (EDF = effective degrees of freedom).

Sibling relationships between jack and adult survival at Bingham Creek were not static through time and varied by thermal regime with lower jack-to-adult ratios observed in cool brood years (1980 to 1999 including La Niña years) compared to warm years (2000 to 2016 including El Niño years). Between brood years 1980 and 2016, coho jack rates ranged from 0.02 to $0.54 \%$ with a significant $\left(p<0.01, R^{2}=0.31\right.$, effective degrees of freedom $[E D F]=1.72$ ) positive shift over time (Fig. 3). Overall, there was little support for the hypothesis that jack rates were affected by variation in freshwater during critical life history phases. However, the top performing GAMM $\left(R^{2}\right.$ $=0.56$ ) determined that jack rates were affected by the thermal regime (cool or warm), Chehalis River flow rates from April to June during outmigration $(k=3)$, El Niño conditions (ONI from January to June of ocean entry; $k=3$ ), and timing of the physical spring transition from predominantly downwelling to upwelling conditions in the year of ocean entry $(k=5$, Fig. 4$)$. While thermal regime was a significant coefficient, it did not change the directional impacts of individual covariates. Jack rates were highest at extreme El Niño values (ONI greater than 0.5 or less than -0.5), when Chehalis River flows were $>2000 \mathrm{cfs}\left(56.6 \mathrm{~m}^{3} \mathrm{~s}^{-1}\right)$ during outmigration, and in years with an earlier spring transition date $(<$ June 1$)$. 

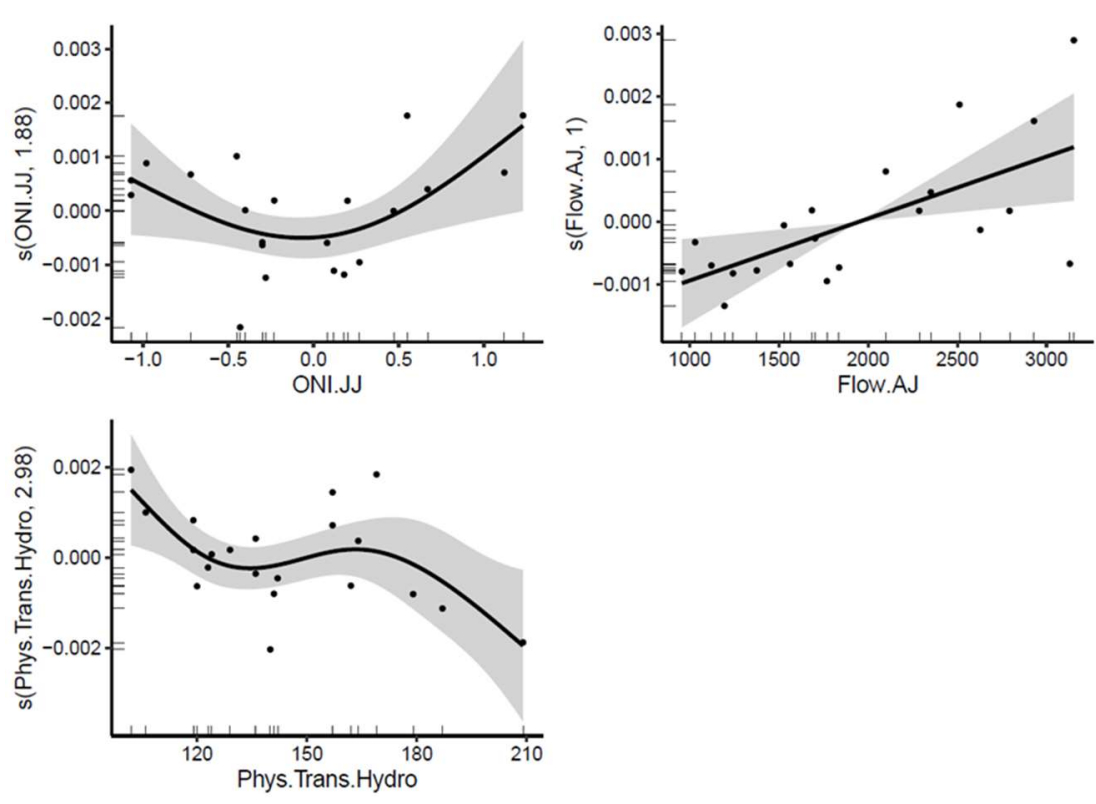

Covariates in the GAMM that best predicted $\left(R^{2}=0.80\right)$ wild adult coho marine survival at Bingham Creek included timing of the physical spring transition from predominantly downwelling to upwelling conditions in the year of ocean entry $(k=4)$, wild coho jack rate from the same brood that returned to the trap the previous year $(k=$ $4)$, and an indicator that integrated information about the North Pacific climate during the summer of ocean entry (ALBSA, $k=4$ ), plus thermal regime (cool or warm, Fig. 5). Unlike the jack model, thermal regime significantly ( $p$ $<0.01)$ affected the directional response of the covariates. However, like jacks, higher adult coho marine survival was associated with earlier spring transition dates $(<$ June 1), but also the ALBSA index, with higher survival observed during lower ALBSA index values $(<50)$. Adult survival was also strongly positively related to jack rate, but during warm regimes only.
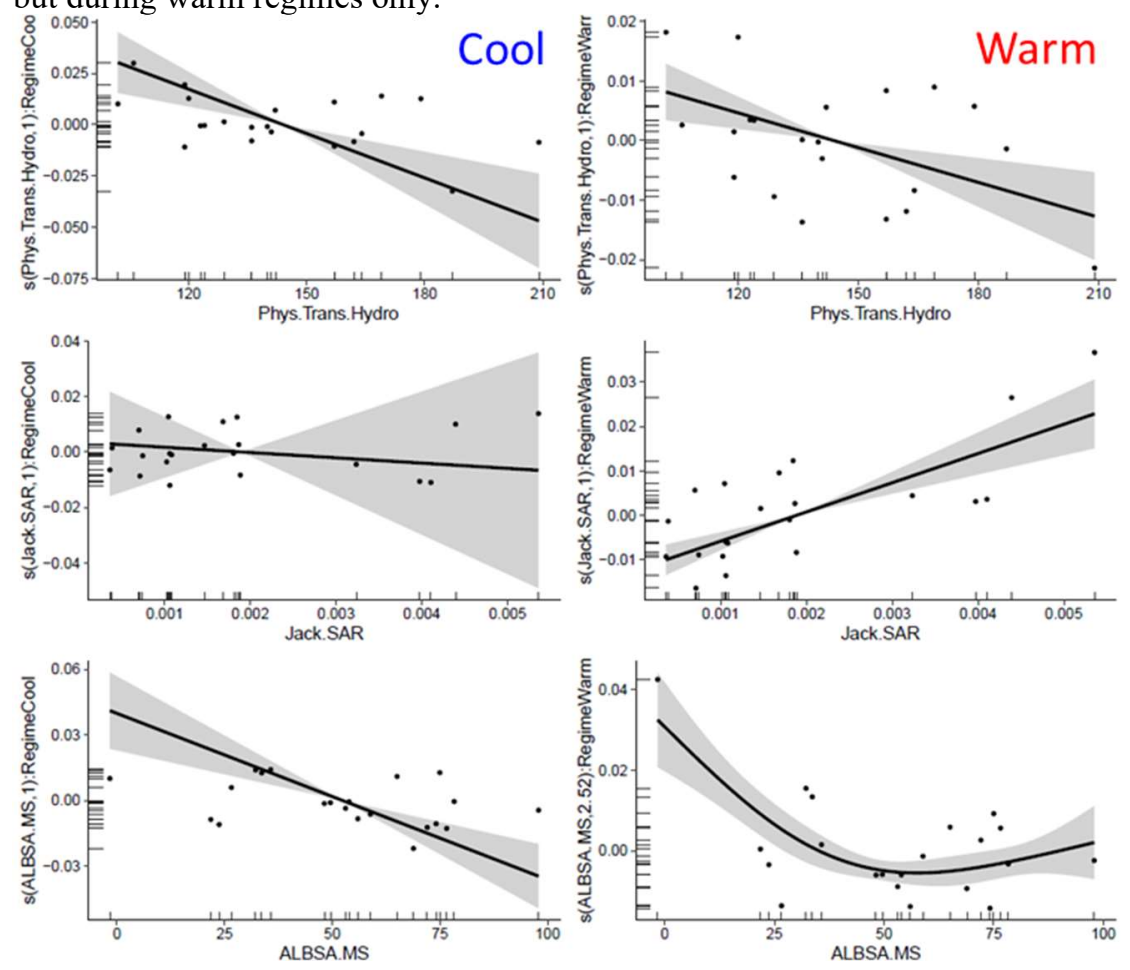

Fig. 4. Top performing generalized additive mixed model predicting Bingham Creek jack rate as a function of thermal regime, the Oceanic Niño Index from January to June (ONI.JJ), stream flow in the Chehalis River from April to June (Flow.AJ), and the physical Spring transition date based on hydrography (Phys.Trans.Hydro) in the year of ocean entry (brood years 1996 to 2016). Note that although significant, the regime coefficient did not lead to variation among the top covariates.
Fig. 5. Top performing generalized additive mixed model predicting Bingham Creek adult marine survival as a function of thermal regime (cool on left and warm on right), the physical spring transition date based on hydrography (Phys.Trans.Hydro), jack rate, and the Aleutian-Low Beaufort Sea Anticyclone index from May through September (ALBSA.MS) in the year of ocean entry (brood years 1996 to 2016). Note that the thermal regime coefficient had varying directional effects on the top covariates. 
Results of one-year ahead forecasting (Table 3 ) found that for the jack model, the GAMM slightly overpredicted abundance (MRE $=-6.6 \%)$. For the adult model, the GAMM also slightly over-predicted abundance $(\mathrm{MRE}=-14.5 \%)$ but was within the acceptable range for forecasting adult returns. The adult model was particularly successful in 2019, a year when many forecasts over-predicted adult returns of wild coho.

Table 3. Statistics of fit for the one-year-ahead models predicting jack abundance and adult returns to Bingham Creek. Model evaluation statistics are mean raw error (MRE), mean absolute error (MAE), root mean square error (RMSE), mean percent error (MPE), and mean absolute percent error (MAPE).

\begin{tabular}{lll}
\hline Statistic & Jack Model & Adult Model \\
\hline MRE & 3.19 & -88.4 \\
MAE & 11.33 & 126.52 \\
RMSE & 15.62 & 159.21 \\
MPE & $-6.6 \%$ & $-14.5 \%$ \\
MAPE & $24.8 \%$ & $26.7 \%$ \\
\hline
\end{tabular}

The expression of the jack life history at Bingham Creek appeared to have two modes based on trade-offs in the coastal marine environment. Under the first mode, jack rates were positively related to flow during the outmigration window and negatively related to the timing of the spring transition. In those years, smolts carried out of the Chehalis River during strong flow regimes were more likely to return as jacks, rather than adults, when the timing of the physical spring transition occurred before June. Earlier spring transition dates are typically associated with higher prey availability, thereby allowing juveniles to reach the critical growth or lipid accumulation threshold required for jacking. However, jack rates also increased over the last decade and a half, and under this model, appeared to be the result of climate variation in the nearshore environment, with warmer periods, including El Niño years, producing more jacks. In those years, jack rates increased during poor ocean conditions and possibly emerged as a trade-off between remaining in an unfavorable ocean versus returning to freshwater to reproduce. Note that jack rates were highest at both high and low ONI values and increased during both favorable (cool, early onset of upwelling) and unfavorable (warm, delayed upwelling) conditions.

No freshwater indicators were identified as being important predictors of jack rate in Bingham Creek for brood years 1996 to 2016. This does not mean that environmental conditions experienced during the freshwater rearing period are not contributing to increased jack rates. There is certainly room to explore other temperature metrics that may not be correlated with mean August stream temperature but could influence behavior, growth, or ecology of juvenile salmon during the freshwater rearing period. By mid-century (2040), temperatures in Bingham Creek are expected to increase by $9 \%$ to $14.5^{\circ} \mathrm{C}$ and by late-century (2080) they will increase by $21 \%$ to $16.0^{\circ} \mathrm{C}$. The main stem Chehalis and many of the major tributaries in the basin are projected to exceed $18^{\circ} \mathrm{C}$ in August by 2080 (Winkowski and Zimmerman 2019), approaching the upper limit of thermal tolerance for juvenile salmonids.

\section{REFERENCES}

Brodeur, R.D., S. Ralston, R.L. Emmett, M. Trudel, T.D. Auth, and A.J. Phillips. 2006. Anomalous pelagic nekton abundance, distribution, and apparent recruitment in the northern California Current in 2004 and 2005. Geophys. Res. Lett. 33: L22S08.

DeFilippo L.B., D.E. Schindler, J. Ohlberger, K.L. Schaberg, M.B. Foster, D. Ruhl, and A.E. Punt. 2019. Recruitment variation disrupts the stability of alternative life histories in an exploited salmon population. Evol. Appl. 12: 214-229.

Isaak, D.J., E.E. Peterson, J.M. Ver Hoef, S.J. Wenger, J.A. Falke, C.E. Torgersen, C. Sowder, E.A. Steel, M.J. Fortin, C.E. Jordan, A.S. Ruesch, N. Som, and P. Monestiez. 2014. Applications of spatial statistical network models to stream data. WIREs Water 1(3): 277-294.

Isaak, D.J., S.J. Wenger, E.E. Peterson, J.M. Ver Hoef, D.E. Nagel, C.H. Luce, S.W. Hostetler, J.B. Dunham, B.B. Roper, and S.P. Wollrab. 2017. The NorWeST summer stream temperature model and scenarios for the western US: A crowd-sourced database and new geospatial tools foster a user community and predict broad climate warming of rivers and streams. Water Resour. Res. 53: 9181-9205.

R Core Team. 2019. R: a language and environment for statistical computing. R Foundation for Statistical Computing, Vienna. www.r-project.org 
Schwing, F.B., N.A. Bond, S.J. Bograd, T. Mitchell, M.A. Alexander, and N.J. Mantua. 2006. Delayed coastal upwelling along the U.S. West Coast in 2005: a historical perspective. Geophys. Res. Lett. 33: L22S01.

Sobocinski, K.L., C.M. Greene, J.H. Anderson, N.W. Kendall, M.W. Schmidt, M.S. Zimmerman, I.M. Kemp, S. Kim, and C.P. Ruff. 2021. A hypothesis-driven statistical approach for identifying ecosystem indicators of coho and Chinook salmon marine survival. Ecol. Indic. 124: 107403.

Winkowski, J., E. Walther, and M. Zimmerman. 2018. Summer riverscape patterns of fish, habitat, and temperature in sub basins of the Chehalis River, 2013-2016. FPT 18-02. Washington Department of Fish and Wildlife, Olympia, WA.

Winkowski, J., and M.S. Zimmerman. 2019. Thermally suitable habitat for juvenile salmonids and resident trout under current and climate change scenarios in the Chehalis River, WA. Final Report to the Office of the Chehalis Basin. Washington Department of Fish and Wildlife, Olympia, WA.

Wood, S.N. 2017. Generalized additive models: an introduction with R, 2nd edn. CRC Press, Boca Raton, LA. 